\author{
Military Technical College \\ Kobry El-Kobba \\ Cairo, Egypt
}

12-th International Conference

on

Aerospace Sciences \&

Aviation Technology

\title{
INFLUENCE OF ERROR RATE IN DIGITAL DATA TRANSFER ON QUALITY OF RECONSTRUCTED IMAGES PREPROCESSED BY JPEG COMPRESSION BASED ON DCT
}

\author{
Abdel-Monem M. El-Mahdy*
}

\begin{abstract}
Compression process in remote sensing confronts the problem of errors encountered in digital data transfer, when reconstructing the original image. A study on DCT bases for JPEG compression process is carried out on a sample image and a typically remote sensed image. A formula for quantization matrix as a function in one variable is proposed. Effective compression ratio $(E C R)$ is investigated versus the variable of this formula. In ideal case of data transfer, ECR can get a value of 18 with root mean square error (RMSE) $<6 \%$. The peak signal to noise ratio (PSNR) is used as a quantitative figure of merit. It's found to be $>55$ for ECR $\leq 19$ for the two selected images.

Data may encounter errors in its transfer process. Therefore, error rate and level are proposed as 2 independent variables to analyze image quality after reconstruction. Error rate is taken to be one faulty bit or a burst of bits for every $8,16,32,64,96$, and $128 \mathrm{Kbit}$ of transferred data. It is found that the quality of reconstructed image is the same like that of ideal data transfer at low error rate $\leq 1 / 100 \mathrm{~K}$. This quality starts to degrade dramatically if the error rate grows beyond $1 / 64 \mathrm{~K}$ as a threshold value, especially at compression ratios ECR $<11$.

The influence of error rate is only investigated for low compression ratios. The errors in transfer of data are hidden underneath the encountered errors by high compression ratios.
\end{abstract}

\section{KEY WORDS}

Remote sensing, Compression, DCT, JPEG, error rate.

\footnotetext{
* Payload group leader of Egyptsat-1

Prof. in Space science dep. NARSS, Cairo, Egypt, <abdelmonem51@yahoo.com>
} 


\section{INTRODUCTION}

Data compression in remote sensing is essential. This is due to the limitation in storage capacity and restrictions imposed on down link data rate. Therefore it is significant to add a compression module to data handling unit installed on the remote sensing satellite (RSS) board.

The implementation of data compression unit has to be preceded by a deep study on the used mathematical algorithm with defined figures of merit to evaluate the compression method at different variants.

Error rate and the amplitude of this error (error level) in the stream of binary data may describe the problems which may be encountered in data transfer.

In this paper the effect of these parameters on root mean square error (RMSE) and peak signal to noise ratio (PSNR), is studied as figures of merit of reconstructed image quality. I llustration of analysis procedure is shown in figure (1). It is carried out on MATLAB environment.

\subsection{Theoretical basis}

Discrete cosine transform (DCT) [1-5] has been selected in this paper as the basis of JPEG transform.

Matrix A reads a square fragment of the image of size $n \times n$. This matrix is transformed by conversion matrix $\mathrm{C}$ considering the following mathematical sequence:

$$
C(i, j)=\sqrt{2 / k n} \cdot \cos [(i-1)(2 j-1) \pi / 2 n]
$$

Where $k=1$ for $i=2: n, j=1: n$, and $k=2$ for $i=1, j=1: n$

The resultant converted matrix is proposed to be

$$
B=C^{*} A^{*} C t
$$

Where $C t$ is the transpose of $C$. So to reconstruct the original image which is represented by matrix $A$, the matrix $B$ has to be operated by the inverses of $C \& C t$ on both sides of equation 2.2 to be:

$$
\begin{aligned}
C i * B^{*} C t i & =C i * C * A * C t * C t i \\
& =A
\end{aligned}
$$

where $\mathrm{Ci}$, Cti are the inverse matrices of $\mathrm{C}$ and $\mathrm{Ct}$ respectively.

Now to complete the sequence of compression, B elements are divided on quantization elements of matrix $Q$. Such a quantization matrix has been optimized to get the best results which are monitored by the highest number of zeros after truncation. In our study, the following equation is optimized:

$$
Q(i, j)=2(i+j-1) q
$$

Where $Q$ is the quantization matrix and $q$ is the matrix number. So after quantizing the DCT transformed matrix, we get a matrix with a remarkable number of zeros. To get maximum number of consequent zeros, we read the resulting matrix in a zigzag form. By this sequence we get a one-dimensional array of reduced number of elements. The reduction of number of elements is got by counting and replacing the number of zeros instead of their values. The effective compression is the ratio of $\left(n^{*} n\right)$ to the number of elements of obtained one dimensional array. 
Carrying out the compression procedure to get a compressed data stream, and adding a prescribed error by a certain rate, then processing inversely on compressed data stream we get the most significant and the nearest image to original but slightly different.

The difference between the two images may be investigated by calculating the root mean square error:

$$
R M S E=\sqrt{\sum_{i=1}^{M} \sum_{j=1}^{N}[a(i, j)-a d(i, j)]^{2} / M * N}
$$

Where $a(i, j) ; a d(i, j)$ are the signal levels of corresponding pixels in original and decompressed images, and $(M * N)$ is the dimension of image fragment under study. Another used evaluation figure of merit is the peak signal to noise ratio which may be expressed as:

$$
P S N R=20 * \log (255 / R M S E)
$$

If packing and transfer of data are ideal as well as unpacking process and having reconstructed the image, its quality and the compression process can be evaluated using formulae (5) and (6).

In this stage the error rate and its level are introduced in simulating transmission of compressed data and its reception and then decompressing it to reconstruct such an image. Consequently RMSE and PSNR would be evaluated as a function of quantization matrix parameter $q$ to be able to discriminate the error effect on reconstructed image quality with respect to original one.

\section{RESULTS}

Having carried out such an algorithm for different images and for a group of quantization matrices we have got the following results.

\subsection{Race image as test sample}

A race image sample is used as a preliminary test with 20 quantization matrices. Figure (2) shows original image and 2 reconstructed images from the compressed data stream compressed using 2 different quantization matrices with $\mathrm{q}=1$ and 20 . The corresponding effective compression ratios (ECR) are $\approx 3.5$ and 15 consequently, figure (3). The (RMSE) is almost limited to $<0.045$ for ECR $<15$ which is reasonable for such type of images. PSNR is also >60 which ensures the capability of used compression algorithm. Calculating the above mentioned three parameters ECR, RMSE, and PSNR for a typical remotely sensed image, the resulting characteristics are plotted versus quantization matrix number (q) in the same figure (3) to serve in comparison between different images. 
Comparing the results of calculating ECR, RMSE and PSNR got for a race sample image and a typically remote-sensed image. We may observe that they have the same trend but they differ slightly in values due to the image properties. If the PSNR is taken to be>60, ECR is limited by 12 .

Corresponding remotely sensed and reconstructed images using quantization matrices of $q=1$ and 20 are shown in figure (4).

\section{ERROR RATE \& LEVEL EFFECT ON DECOMPRESSED I MAGE QUALITY}

Consider that an error is generated in digital data transfer in packing, communication link of data transfer, unpacking or any other processes before decompression and image reconstruction.

\subsection{Error Rate Effect}

If it is proposed that the stream of data has one faulty bit for each transferred 128, 32, 16, 8 Kbit. The RMSE \& PSNR of such analysis are shown in figure (5).

If the error rate is $<1 / 128 \mathrm{~K}$ the effect on image quality after compression is negligible. But it is remarkable when the error rate is in the order of $1 / 32 \mathrm{~K}$. Therefore in remote sensing, it is preferable to be one faulty bit per 100K.

\subsection{Error Level Effect}

In these analyses the effect of this faulty bit is intentionally considered as least significant bit (LSB). The error level changes from 1:128 according to the position of the faulty bit in the 8-bit word. The value of RMSE is affected dramatically specifically in high error rates. This idea is clearly illustrated for RMSE, in figure (6).

For small error rates $<1 / 64 K$, the effect of error level can be neglected. As shown in figure (7), the RMSE is effectively the same for the error level varieties from 1 to 128 especially for high ECR.

\subsection{Limits on Error Rates}

When fixing the error level and calculating RMSE for different rates it is possible to investigate the combined limits which should be imposed on error rate in digital signal processing or communications.

It's obvious from figure (8) that the value of $1 / 64 \mathrm{~K}$ may be considered as a threshold limit for acceptable image quality. The error level affects only in the higher rate of errors and dramatically beyond rates of $1 / 16 \mathrm{k}$.

In the following part of this work a group of reconstructed images is presented to show the effect of low error rate as well as the burst size effect, figure (9). 


\section{CONCLUSION}

- The RMSE doesn't exceed 0.05 if PSNR has to be kept $>60$. This limits the JPEG compression with DCT basis to ratios of ECR $<12$.

- Error rate of one bit per $10^{5}$ is quietly ineffective for ECR $>8$, if the error bit is LSB but it may be effective for bursts covering byte length.

- Dramatic increase in RMSE is observed for error rate $>1 / 32 \mathrm{~Kb}$

- The error rate is effective in DCT basis JPEG compression for our proposed quantization function only for effective compression ratios $<\sim 11$.

\section{REFRENCES}

[1] W.B. Pennebaker, J.L. Mitchell, "The JPEG Still Image Data Compression Standard", Van Nostrand Reinhold, 1993.

[2] Subhasis Saha "Image Compression - from DCT to Wavelets: A Review", ACM org. Jan.,2001 www.acm.org/crossroads/xrds6-3/sahaimgcoding.html

[3] Zarlink semiconductors, "An overview of the H.261", Application note, AN 206-1.1, Oct., 1995, $<$ www.zarlink.com>.

[4] Li.Z, X.Yuan, and K.Lam; Effect of JPEG compression on the accuracy of photogrammetry point Determination.vol.68, No.8,pp.847-853, 2002

[5] Li\&Drew, "Fundamentals of Multimedia", Image comp. standards, chap.9, Prentice Hall, 2003.

\section{Acknowledgement}

I would like to appreciate too much the sincere effort done by the member of my payload group Engineer Ayman Mahmoud, and the remarks done by my colleague professor Matar A. Matar the leader of communication group in Egyptsat-1 satellite.

\section{FIGURES}

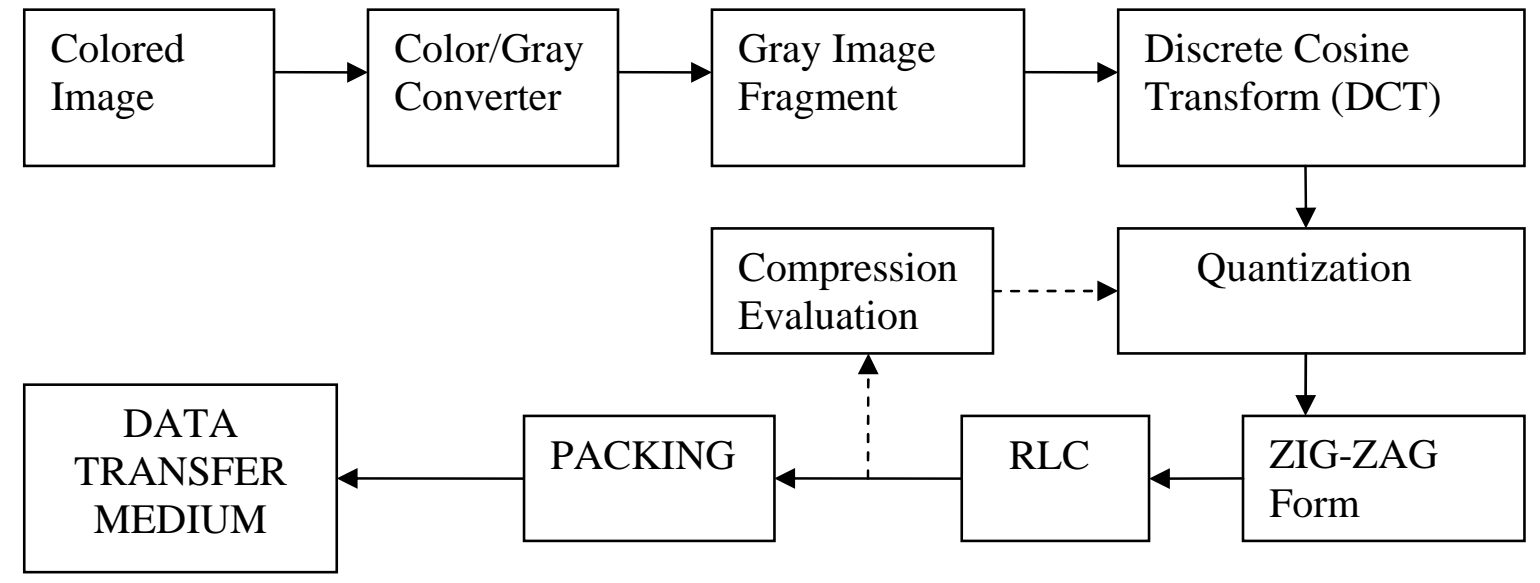

Figure (1) Procedure of image fragment compression until packing and data transfer to ground reception station. 


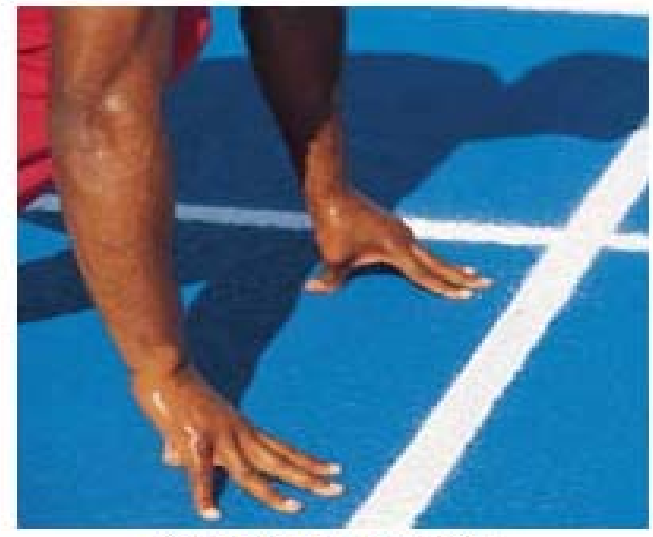

ORIGINAL IMAGE

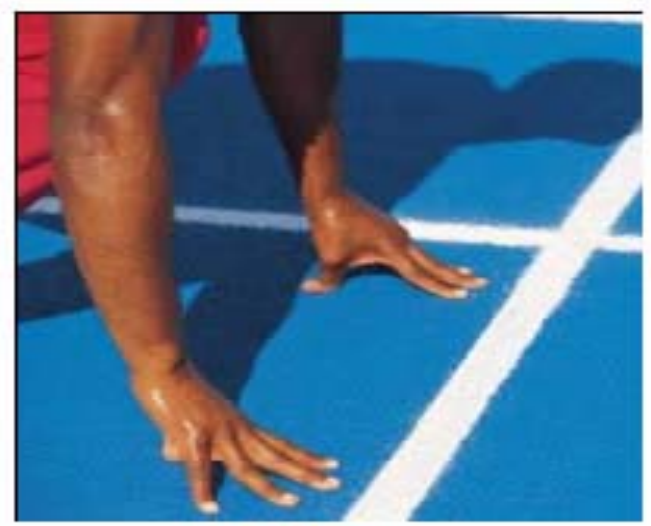

MATRIX 1

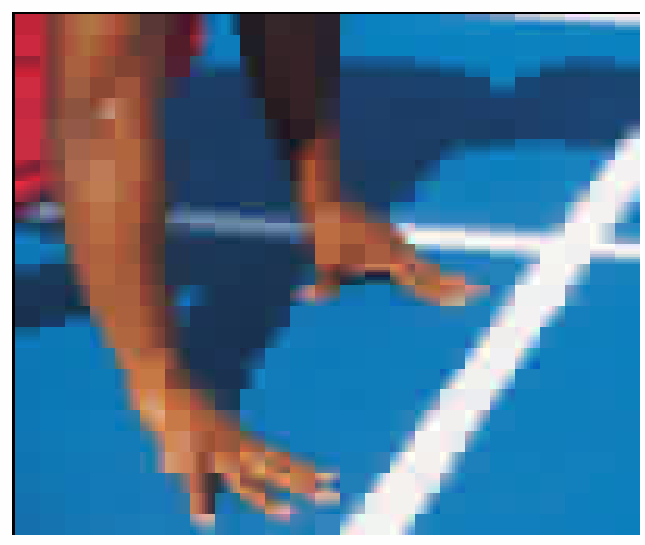

MATRIX

Figure (2) Test image and 2 decompressed images with different compression ratios.
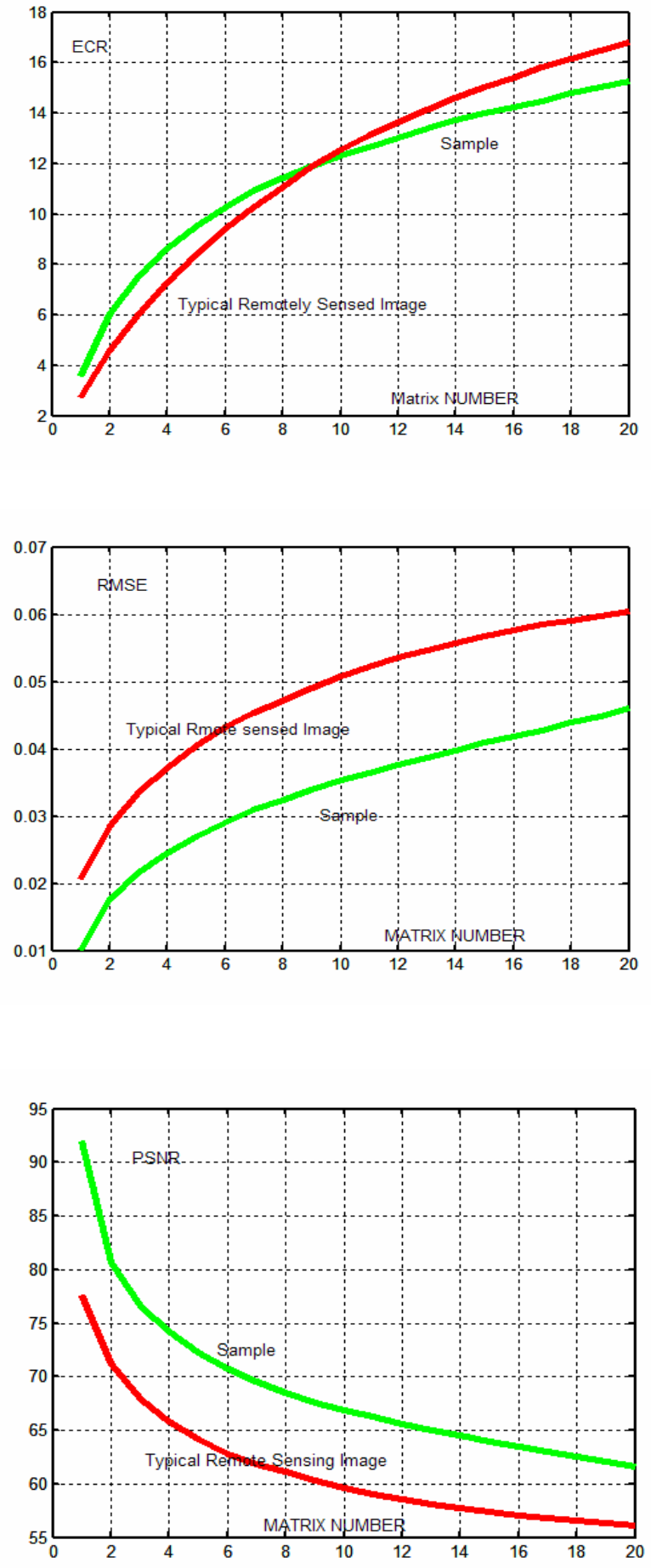

Figure (3) ECR, RMSE and PSNR versus quantization matrix number(q) for race sample and a remotely sensed images. 




ORIGINAL IMAGE

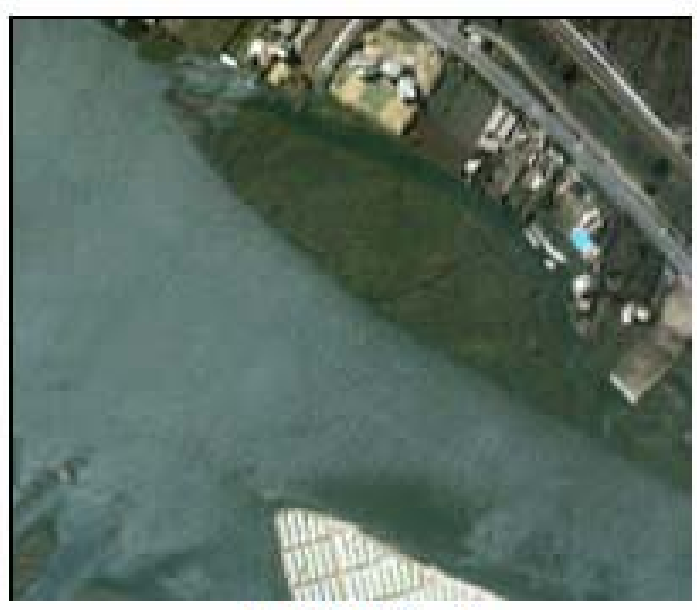

MATRIX 1

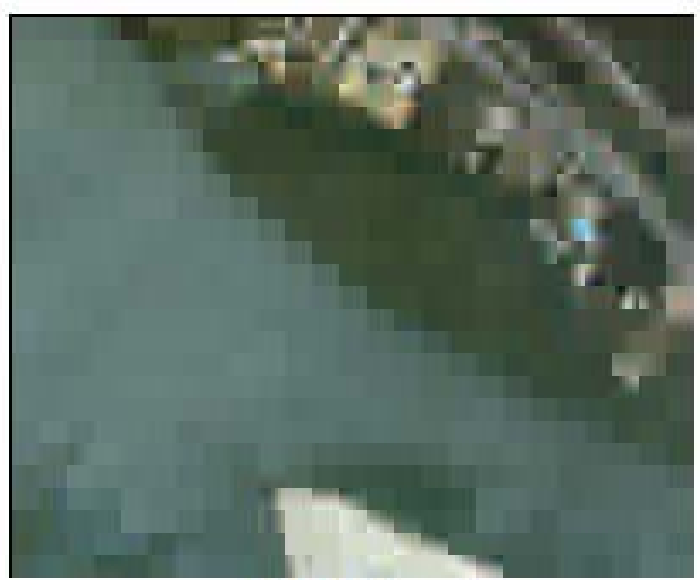

MATRIX $\quad 20$
Figure (4) typical remotely sensed image and corresponding 2 decompressed images with different compression ratios.

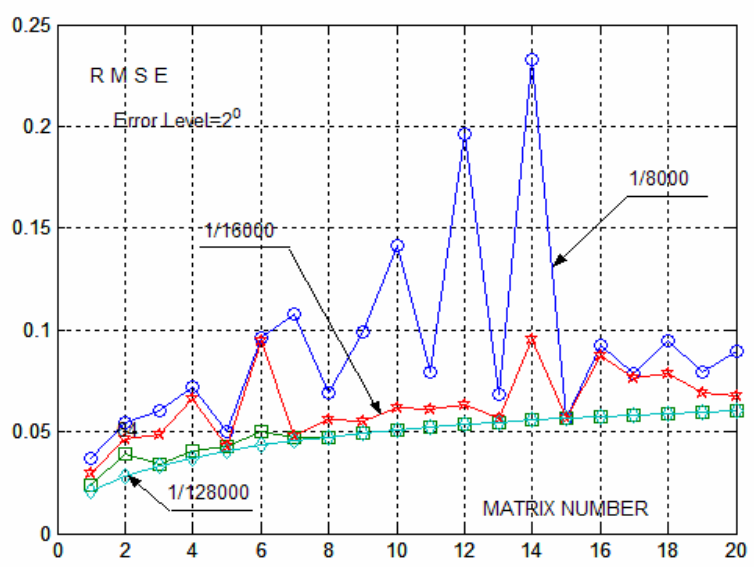

Figure (5) RMSE variation v-s quantization matrix number for different error rates of $1 / 128000$, $1 / 32000,1 / 16000$, and $1 / 8000$ at error level $2^{\wedge} 0$.

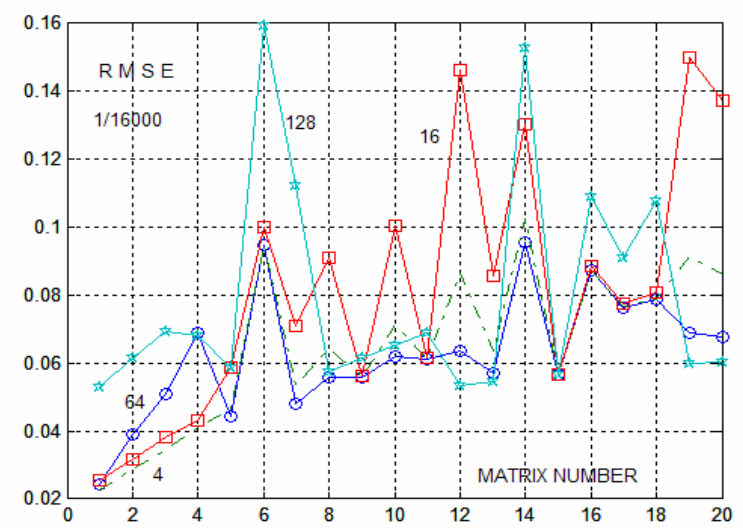

Figure (6) RMSE v-s quantization matrix number for different error levels 4, 16, 64 and 128 at error rate of $1 / 16000$. 

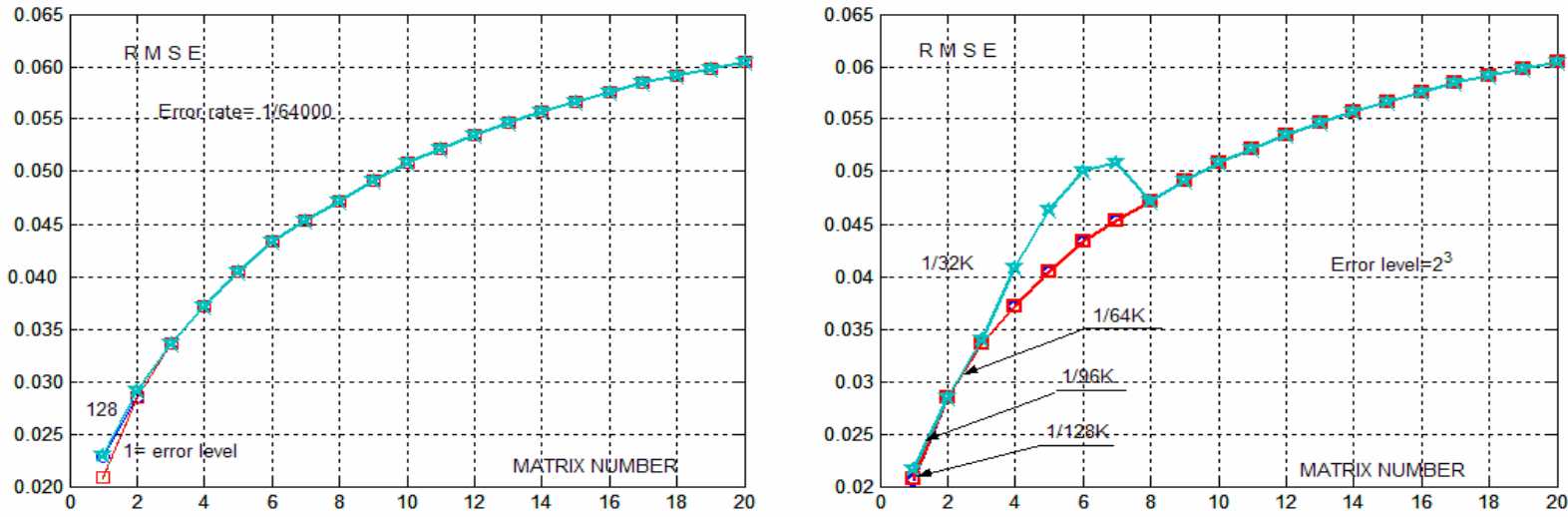

Figure (7) error level affects negligibly at small error rate.
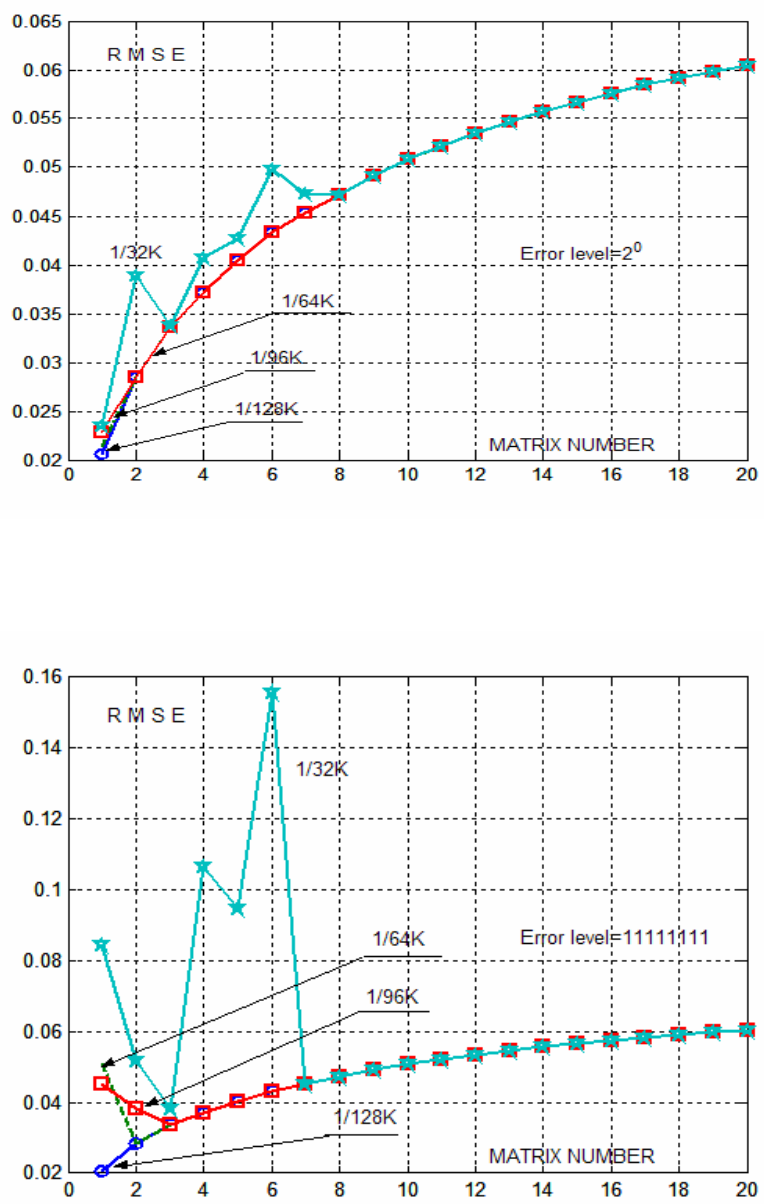

Figure (8) Combined effects of error rate and error levels define the threshold error rate for acceptable image quality. 
(a)

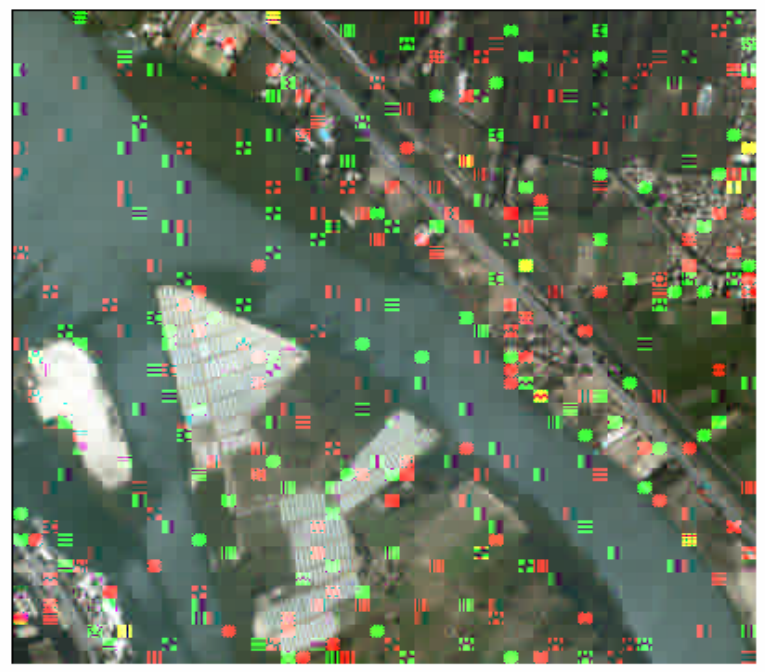

BURST OF ONE FAULTY BIT/320

(b)



BURST OF ONE FAULTY BIT/3200 (c)

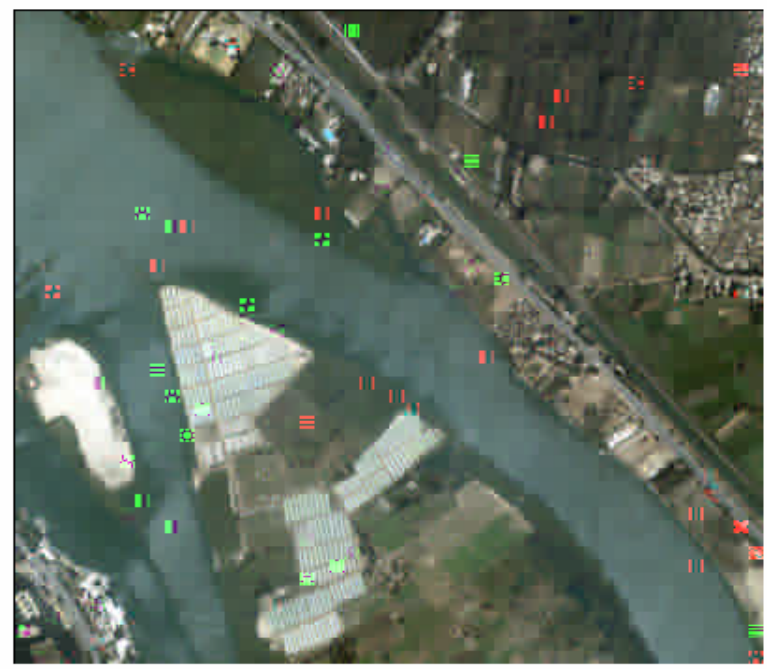

BURST OF FAULTY $(00000101) / 3200$

(d)

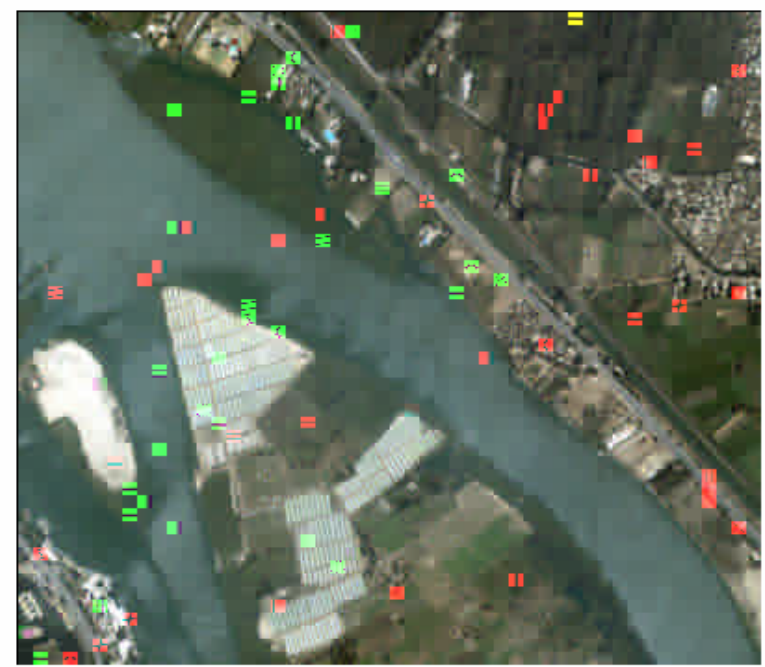

BURST OF FAULTY $(10000000) / 3200$

Figure (9) A group of reconstructed images from compressed data $q=6$. They show the effect of increased rate (a and b), meanwhile the images ( $c$ and d) show the effect of power of faulty bits for the same rate. 